\title{
Transplantation of mononuclear cells from bone marrow in a rat model of Huntington's disease
}

This article was published in the following Dove Press journal:

Journal of Neurorestoratology

13 December 2016

Number of times this article has been viewed

\section{Teresa Serrano' \\ Paula Pierozan ${ }^{2}$ \\ Esteban Alberti' \\ Lisette Blanco' \\ Karelys de la Cuétara \\ Bernal' \\ María E González' \\ Nancy Pavón' \\ Lourdes Lorigados' \\ María A Robinson- \\ Agramonte' \\ Jorge A Bergado'}

'International Center for Neurological Restoration (CIREN), La Habana, Cuba; ${ }^{2}$ Department of Biochemistry, Instituto de Ciências Básicas da Saúde, Universidade Federal do Rio Grande do Sul, Porto Alegre, Rio Grande do Sul, Brazil
Correspondence: Jorge A Bergado Centro Internacional de Restauración Neurológica, Avenue 25, Number 15805, Cubanacán, Playa CP II 300, La Habana, Cuba

Tel +53727 I 5353

Fax +537336028

Email bergado@neuro.ciren.cu
Abstract: This article investigates the possible effects of transplantation of mononuclear bone marrow cells (mBMCs) to ameliorate or prevent the behavioral impairments and the cellular damage observed in a quinolinic acid (QA) model of Huntington's disease. mBMCs were isolated using a standard procedure and implanted within the QA-lesioned striatum. Behavior was explored using motor (beam test) and memory (object recognition and Morris water maze) tests. Morphology was evaluated using conventional histology (cresyl violet), bisbenzimide (to evaluate cell vitality), and immunohystochemistry to identify neurons or glia. mBMC-transplanted animals showed improvements in motor coordination (beam test). Regarding memory, object recognition was significantly improved in transplanted animals, while spatial memory (Morris water maze test) was not severely affected by QA and, therefore, the results after transplantation were significant only in the probe-trial retention test. In samples taken from the animals that participated in the behavioral tests, a preserved morphology of striatal neurons and a reduced glial reaction indicated a possible neuroprotective effect of the transplanted mBMCs. A parallel study confirmed that the transplanted mBMCs have a long survival period (1 year follow-up). The results presented confirm the possibility that $\mathrm{mBMC}$ transplantation may be a viable therapeutic option for Huntington's disease.

Keywords: mononuclear bone marrow cells, Huntington's disease, quinolinic acid, transplant, Fluoro-Jade C, glial fibrillary acidic protein, neuronal nuclear marker

\section{Introduction}

Huntington's disease (HD) is an inherited neurodegenerative disease characterized by motor dysfunction, cognitive decline, and psychiatric disorders. ${ }^{1}$ The striatum is the major site of HD degeneration ${ }^{2}$ and is characterized by a marked loss of the striatal medium-sized spiny projection neurons. ${ }^{3}$ As no cure is currently available, management of symptoms is the primary goal in treating HD.

Some therapies aimed at modifying the course of the disease have been evaluated in animal models, eg, pharmacological interventions and modulation of autophagy targeting pathogenic pathways, while cell replacement therapies that attempt to replace dysfunctional or dying cells, primarily through transplantation, have also been tested., ${ }^{4,5}$ Cell therapy strategies in HD have traditionally been aimed at protecting or replacing cells lost during the course of the disease and thereby preventing or retarding disease progression.

Different animal models that replicate the HD pattern of degeneration have been used to study the pathophysiology ${ }^{6}$ and the treatment ${ }^{7}$ of HD. Quinolinic acid (QA) is 
an excitotoxic substance that, when injected into the striatum, induces death of the projecting neurons. The injection of QA into the striatum has been, since long, used as an animal model of HD. ${ }^{8,9}$

Transplantation of mononuclear bone marrow stem cells (mBMCs) has been successfully used in animals after traumatic brain damage ${ }^{10}$ and striatal ischemia ${ }^{11}$ to reduce the motor deficits induced by the injury.

In a previous study, we have shown that the transplantation of mBMCs to unilaterally QA-lesioned rats reduces the number of turns in the classic amphetamine-induced circling locomotion behavior and facilitates the acquisition of a motor skill. ${ }^{12}$ More recently, we have reported an increase in levels of brain-derived neurotrophic factor (BDNF), both mRNA and protein, measured in the striatum of QA-lesioned rats after mBMC transplantation. ${ }^{13}$

These findings support the idea that the transplanted cells contribute to alleviating the negative symptoms of the striatal lesion and might probably also restore the cellularity within this nucleus of the basal ganglia. In this study, we attempt to complement and expand these previous reports in 3 aspects: first, we aimed to study the effects of this transplantation on motor coordination and memory using behavioral models; the second goal was to obtain a preliminary insight into how the transplantation of mBMCs influenced the cellular composition of the lesioned striatum; and the third aim was to determine the survival of the transplanted cells in the long term.

\section{Materials and methods} Animals

Adult male Sprague Dawley (SD) rats (total N=71) obtained from the National Center for Laboratory Animals Production (CENPALAB) and weighing 200-250 g (between 9 and 12 weeks of age) were used in this study. Animals were housed in translucent macrolon cages ( 5 animals per cage) under 12 hour light:12 hour darkness cycle and ad libitum access to water and food. Experiments were carried out in accordance with the Cuban Regulations for the Use of Laboratory Animals (CENPALAB 1997) and were approved by the Ethical Committee of the International Center for Neurological Restoration. Efforts were made to minimize the pain and discomfort of the animals, as well as the number of aniimal used for experiments.

To characterize the histological conditions in the striatum after QA lesioning at the time of transplantation, a group of 10 animals was sacrificed 1 mo after the lesioning, and the brains extracted and processed by conventional (cresyl violet) histology and glial fibrillary acidic protein (GFAP) immunohistochemistry.
Experimental groups for behavior and morphology included 4 groups $(\mathrm{n}=10$ each) according to treatment: Control (C); QA lesion (QAL); mononuclear bone marrow cell transplantation (mBMC); and Dulbecco's Modified Eagle's Medium injection (DMEM).

The cell survival study was carried out in a separate group of $\mathrm{mBMC}(\mathrm{N}=3$ per group) sacrificed at 10,30 , $45,60,90,180$ days, and 1 year posttransplantation with mBMCs.

\section{Lesioning and transplantation Striatal QA lesions}

Unilateral lesions of the right striatum were produced by the intrastriatal injection of QA. Rats were anesthetized with an intraperitoneal injection of chloral hydrate $(420 \mathrm{mg} / \mathrm{kg}$ body weight). QA injections were administered with the help of a stereotaxic apparatus (model 900; David Kopf Instruments, Tujunga, CA, USA). Each rat was injected in the striatum with $1.2 \mu \mathrm{L}$ of QA (125.5 nmol) (Sigma, Saint Louis, MA, USA) using a $30 \mathrm{G}$ Hamilton syringe at the following coordinates: $1.2 \mathrm{~mm}$ anterior, $2.8 \mathrm{~mm}$ lateral to bregma, and $5.5 \mathrm{~mm}$ below the dura. The toxin was injected over a period of 1 minute, and the cannula was left in place for an additional 10 minutes before being slowly removed.

\section{Obtaining rat bone marrow}

Male SD rats between 32 and 48 days old were anesthetized with an intraperitoneal injection of chloral hydrate (420 mg/kg body weight) and a cut on the skin of the hind limbs was performed, separating the tissue parallel to the bone to extract both femurs; then the animals were euthanized with a lethal overdose of chloral hydrate. The extracted bones were placed for 30 minutes on a Petri dish containing $0.9 \%$ physiologic saline, after which the bone marrow was obtained by flushing with sterile phosphate-buffered saline (PBS; $\mathrm{NaCl}$, $8 \mathrm{~g} / \mathrm{L} ; \mathrm{KCl}, 0.2 \mathrm{~g} / \mathrm{L} ; \mathrm{Na}_{2} \mathrm{HPO}_{4}, 1.09 \mathrm{~g} / \mathrm{L} ; \mathrm{KH}_{2} \mathrm{PO}_{4}, 0.26 \mathrm{~g} / \mathrm{L}$, $\mathrm{pH}$ 7.2) through one of the femoral epiphyses. The bone marrow was collected in sterile containers to be later washed by centrifugation. ${ }^{12}$

\section{Isolation of mononuclear cells from rat bone marrow}

The suspension of bone marrow cells was washed 3 times with $1 \times$ PBS by centrifugation for 10 minutes at $2000 \mathrm{rpm}$ at $20^{\circ} \mathrm{C}$. An aliquot of $2.5 \mathrm{~mL}$ of Ficoll-Hypaque was placed on the bottom of a graduated glass tube, on top of which $5 \mathrm{~mL}$ of the cellular suspension in PBS was layered. This was centrifuged for 45 minutes at $2800 \mathrm{rpm}$ at $20^{\circ} \mathrm{C}$. 
The mononuclear cell band was extracted with a pipette and washed immediately, discarding the supernatant into a container with hypochlorite and collecting the cellular pellet, which was suspended in $1 \times$ PBS.${ }^{14}$ These cells have been previously well characterized in our laboratory ${ }^{15}$ and contain cells positive for the cluster of differentiation (CD)-34, CD38, CD45, and CD90 markers of adult bone marrow stem cells.

\section{Transplantation}

Four weeks after the QA lesioning, the animals to be transplanted (mBMC) were deeply anesthetized as previously described. Rats were placed in the stereotactic apparatus and the skin over the skull was reopened. Using a Hamilton syringe, the mBMC suspension (50000 cells/ $\mu \mathrm{L}$ in DMEM) was injected into the lesioned striatum (in 2 deposits; $1 \mu \mathrm{L}$ per deposit) at coordinates slightly different from the ones used for the QA lesions: $0.7 \mathrm{~mm}$ anterior from the bregma, $2.8 \mathrm{~mm}$ lateral from the midline, and 5.5 and $4.6 \mathrm{~mm}$ under the dura surface. Cells were injected slowly over a period of 1 minute. The needle was left in place for an additional 10 minutes following injection and then carefully removed. Sham-transplanted animals (DMEM) received an equal volume of tissue culture medium injected in the same way, at the same stereotactic coordinates.

To assess the viability of the transplanted cells, a sample of the mBMCs was marked with the reagent Hoechst 33258 $(1 \mu \mathrm{g} / \mathrm{mL})$ in DMEM and incubated for 24 hours at $37^{\circ} \mathrm{C}$ with $5 \% \mathrm{CO}_{2}$. Next, the cells were washed with PBS and cell viability was determined by trypan blue exclusion.

\section{Behavioral tests}

\section{Transversal beam tests}

The transversal beam tests were carried out in a noise-isolated and well-illuminated room. The quantitative measures of the animal's behavior were obtained using a video tracking system (Panlab, Barcelona, Spain) and analytical software
(Smart 2.00) from the same company. A video camera, mounted in the center above the transversal beam apparatus, provided a picture of this on a computer monitor. The rats were placed at the middle point of a beam at a height of $60 \mathrm{~cm}$ from a supporting surface, equipped with escape platforms at each end (Figure 1A). The Smart system analyzes the tracking of the animals on the beam. From this, the total length (in $\mathrm{cm}$ ) traveled by the animal during the video recording (traveled distance) was measured for a period of 60 seconds. The beams were all $60 \mathrm{~cm}$ long but varied in cross-sectional shape and width. The shape of the cross section could be rectangular (RCS) or circular (CCS), with a width of $2.5 \mathrm{~cm}$ or $1 \mathrm{~cm}$. Accordingly, the following 4 combinations were tested: rectangular $2.5 \mathrm{~cm}$ diameter, circular $2.5 \mathrm{~cm}$ diameter, rectangular $1 \mathrm{~cm}$ diameter, and circular $1 \mathrm{~cm}$ diameter. The complete experiment was performed over 2 consecutive days, carrying out 2 assays per day; equivalent to 4 trials for each beam. The final traveled distance was computed as the mean of the 4 values obtained from all assays for each beam type. ${ }^{16}$

\section{Object recognition test}

The test was performed in a square wooden box $(50 \times 50 \mathrm{~cm})$ with walls of $60 \mathrm{~cm}$ height. On the first day, 2 objects of identical form, color, and size were placed in positions $A$ and B (Figure 1B). In each trial, 1 animal was placed in the center of the box, near the wall, and with back to the objects. The animals were allowed to move freely for 3 minutes and the time (in seconds) spent exploring each object was measured. On the second day, the object at location B was replaced by a new object that differed in size, shape, and color. The time spent by the animal exploring each object was measured again. ${ }^{17}$

\section{Morris water maze test}

Animals were trained in the Morris water maze (Figure 1C). In each trial, the rat was introduced into the water, starting
A

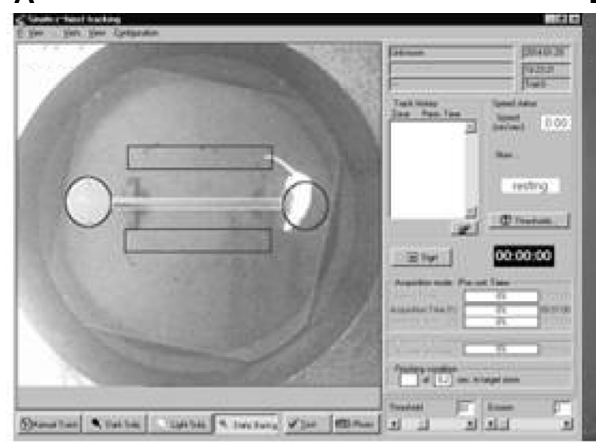

B

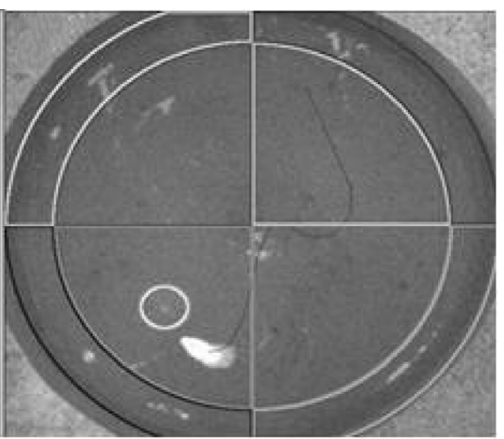

Figure I Photograph of each behavioral test.

Notes: (A) Transversal beam with escape platform; (B) object recognition test; and (C) Morris water maze test. 
randomly from 4 different positions (named arbitrarily using cardinals) and was allowed to search for a hidden escape platform for a period of 60 seconds. If the animal was unable to find the platform, it was gently carried onto it. A 30 second rest period sitting on the platform was allowed between trials. Each training day consisted of 8 consecutive trials, except for the fourth day, in which only 4 trials were carried out. Before the fifth trial (probe trial) the platform was removed, and the animal was allowed to search for it for a period of 60 seconds. The number of crossings over the place where the platform used to be was counted during this trial. ${ }^{18}$

\section{Morphology}

At the completion of behavioral testing, the rats were deeply anesthetized with an injection of $7 \%$ chloral hydrate and transcardially perfused with $0.9 \%$ saline followed by $4 \%$ paraformaldehyde. The brain was removed and postfixed for 2 hours in $4 \%$ paraformaldehyde at $4^{\circ} \mathrm{C}$. The tissue was then successively transferred into $7 \%, 15 \%$, and $30 \%$ sucrose solutions in potassium-sodium phosphate buffer at $4{ }^{\circ} \mathrm{C}$. After the brain sank (2-3 days), it was frozen by immersion in isopentane, cooled with $\mathrm{CO}_{2}$, and stored in a freezer $\left(-80^{\circ} \mathrm{C}\right)$ for later analyses. Serial coronal sections $(20 \mu \mathrm{m})$ of the striatum were obtained using a cryostat at $-20^{\circ} \mathrm{C}$ (Leica). A set of 6 sections, taken from the same region in all groups throughout the rostrocaudal axis of the dorsal striatum, were used for cresyl violet and histochemical analysis.

\section{Cresyl violet}

This basic stain was used to show the general cytoarchitecture of the striatum. Briefly, the slices were placed for 2 hours in absolute alcohol at room temperature, followed by 1 hour exposure to a cresyl violet solution. The excess stain was washed in water, subsequently dehydrated in rapid transfers of absolute alcohol, and finally cleared in xylol. The slices were mounted on slides to be observed under an Olympus SR-2 (Tokyo, Japan) conventional microscope.

\section{Immunofluorescence and Fluoro-jade C staining}

Brain slices from animals of all trained groups were obtained from the region of transplantation. These sections were washed several times in PBS, transferred to gelatinized slides, and incubated 48 hours with rabbit polyclonal anti-GFAP or mouse anti-neuronal nuclear marker (anti-NeuN) (clone A60) antibodies, diluted 1:3000 and 1:1000, respectively, in PBS containing $0.3 \%$ Triton X-100 (PBS-Triton X-100 0.3\%) and $2 \%$ bovine serum albumin (BSA $2 \%$ ). The negative controls were set up omitting the primary antibodies. After washing several times in PBS, the tissue sections were incubated with anti-rabbit or anti-mouse $\mathrm{Cy} 3$ antibodies, both diluted 1:500 in PBS-Triton X-100 0.3\% and BSA 2\% for 1 hour at room temperature. Afterward, to visualize degenerating neurons, the slides were air-dried and subjected to Fluoro-Jade ${ }^{\circledR} \mathrm{C}$ (FJC) staining according to a previous report, ${ }^{19}$ with slight modifications. Briefly, slides were rinsed for 5 minutes in distilled water and then incubated in $0.06 \%$ potassium permanganate solution for 10 minutes. Following a 2 minute water rinse, slides were incubated for 10 minutes in the FJC staining solution with $0.001 \%$ 4',6-diamidino-2-phenylindole (DAPI). The slides were washed, dried, cover-slipped in acidic mounting medium (distyrene plasticizer xylene), and examined under an epifluorescence microscope. The images were obtained with an Olympus IX-81 confocal FV-1000 microscope and analyzed with the Olympus Fluoview software.

\section{Cell survival study}

To evaluate the survival of the transplanted cells, a separate group of untrained $\mathrm{mBMC}$ animals were sacrificed at different moments posttransplantation: ( $\mathrm{n}=3$ each time) on days 10 , $30,45,60,90$, and 180, as well as at 1 year. These animals did not participate in the behavioral study. The extracted brains were processed as described in the previous section and 3 series of slices were collected to carry out the observation. In all the cases, the striatum was sectioned in $20 \mu \mathrm{m}$ slices using as reference the anterior commissure according to the stereotactic atlas. ${ }^{20}$ The Hoechst 33258-labeled cells were then detected by their fluorescence microscopy $(\lambda=420 \mathrm{~nm}$; Olympus BX51, DM400).

Figure 2 provides a schematic view of the experimental protocol.

\section{Statistical analysis}

All data are expressed as mean values \pm SEMs. Statistical significance of differences for group comparisons was assessed by analysis of variance (ANOVA). Post hoc comparisons were performed using the Tukey's honest significant differences (HSD) test. Differences between groups were considered to be significant when $p<0.05$.

\section{Results \\ Striatal QA lesion}

Figure 1A shows a coronal section at the striatal level where QA (125.5 nmol) was injected into the right striatum (asterisk). QA causes neuronal death and structural alterations. Notice the marked dilatation (arrow) of the lateral ventricle in the side of lesion when compared with the contralateral side. 
Lesion, transplant, behavior, and morphology study

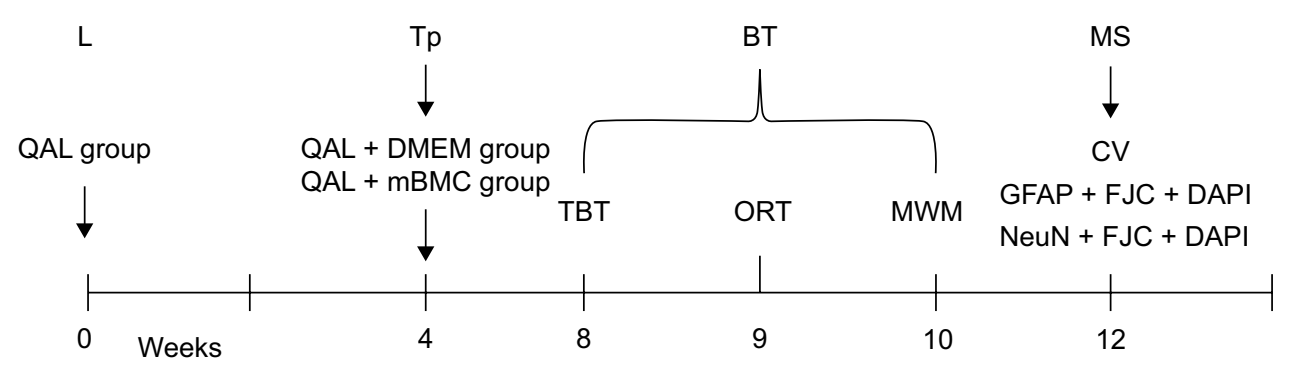

Cell survival study

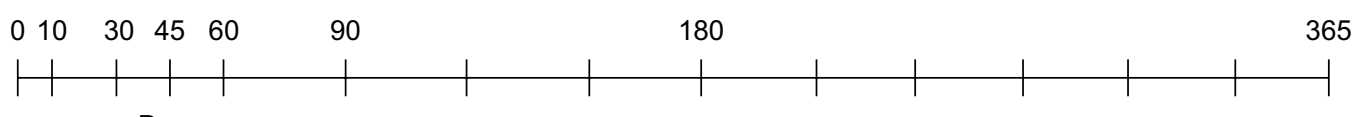

Days

Figure 2 Schematic view of the experimental protocol.

Notes: Male Sprague Dawley rats ( $56-63$ days old), were housed in cages (dimensions: $59 \times 38.5 \times 20 \mathrm{~cm}, 5$ rats in each cage). Chemical lesions were made by the injection of QA ( $125.5 \mathrm{nmol}$ in $2 \mu \mathrm{L}$ PBS) I week later. Rats received a transplant of mBMCs ( 50000 cells $/ \mu \mathrm{L})$ into the lesioned striatum (in 2 deposits; I $\mu \mathrm{L}$ per deposit) I month later. For the behavioral evaluation, a TBT, an ORT, and an MWM test were carried out at 8, 9, and 10 weeks after mBMC transplantation, respectively. After completion of the behavioral tests (Week 12), the animals were sacrificed for the morphology studies. CV staining and immunofluorescence with rabbit polyclonal anti-GFAP or mouse antiNeuN and Fluoro-Jade C (FJC) staining was conducted using coronal sections $(20 \mu \mathrm{m})$ stereologically prepared following fixation. Consecutive sections were used for CV, GFAP + FJC staining, or for NeuN + FJC staining. The cell survival study was carried out at different times after transplant in a separate experiment.

Abbreviations: BT, behavioral testing; CV, cresyl violet; DAPI, 4',6-diamidino-2-phenylindole; DMEM, Dulbecco's Modified Eagle's Medium; GFAP, glial fibrillary acidic protein; L, lesion; mBMC, mononuclear bone marrow cell; MS, morphology study; MWM, Morris water maze; NeuN, neuronal nuclear marker; ORT, object recognition test; PBS, phosphate-buffered saline; QA, quinolinic acid; QAL, QA lesion; TBT, transversal beam test; Tp, transplant.

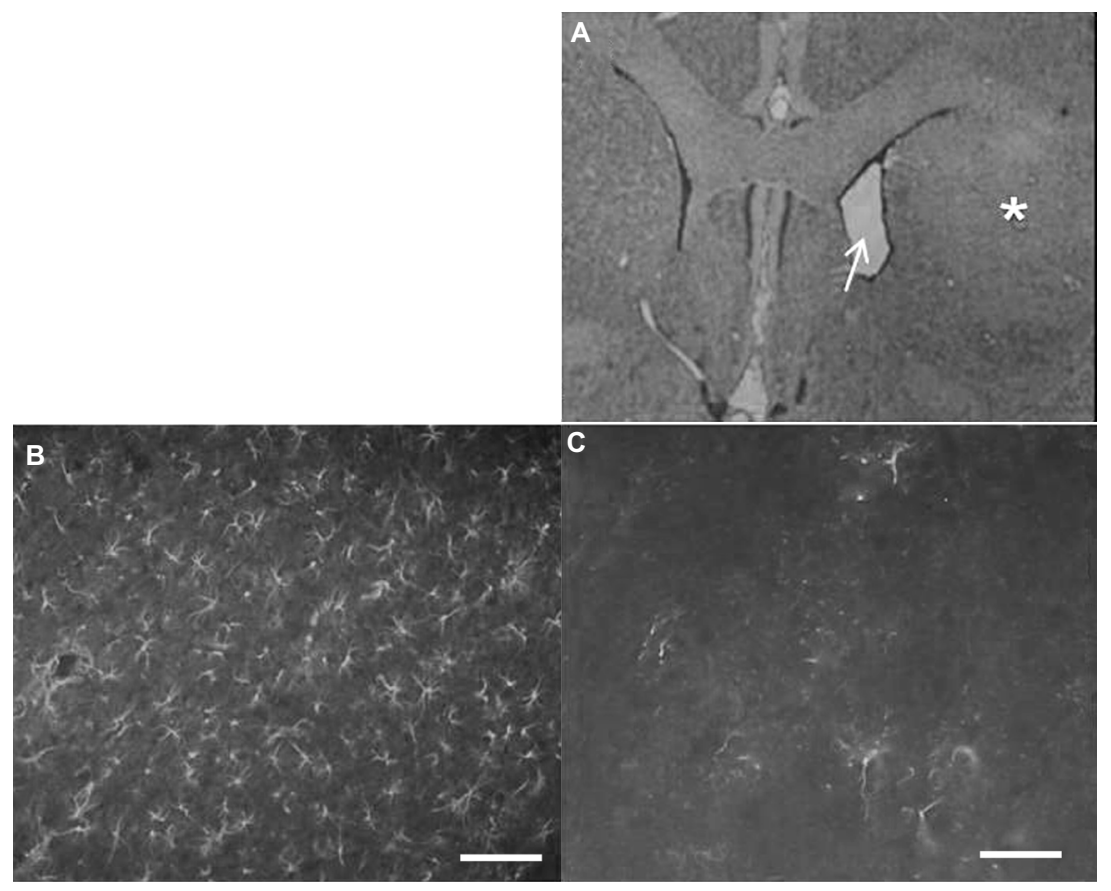

Figure 3 Morphological study of the quinolinic acid (QA) lesion.

Notes: (A) Coronal section from the brain of QA-lesioned rat. Notice the reduced volume of the lesioned striatum (asterisk) when compared with the nonlesioned side (left). This difference is also evidenced by the expansion of the lateral ventricle (arrow) in the lesioned side (200x). (B) Profuse GFAP staining in the lesioned striatum indicating an intense astrogliosis as a consequence of neuronal death when compared with (C) immunohistochemistry for GFAP in nonlesioned striatum. Scale bars: $20 \mu m$. Abbreviation: GFAP, glial fibrillary acidic protein. 
Immunohistochemistry for GFAP shows intensive astrogliosis in the lesioned striatum (Figure 3B) when compared with the contralateral side (Figure 3C). This represents the condition of the striatal tissue at the time when the transplantation of mBMCs was performed.

\section{Behavioral tests}

\section{Transversal beam test}

The transversal beam test was performed 1 mo after transplantation with mBMCs. A factorial ANOVA demonstrated that the variables "Group" and "Beam" significantly influence the distance traveled on the beam. The size and shape of the beam (factor Beam) significantly influence the results; as the test difficulty is increased (width decreased and roundshaped beam), the results worsen $\left(F_{3,167}=6.16, p<0.001\right.$; Figure 4). Between-group differences were observed in this task $\left(F_{3,167}=8.52, p<0.001\right)$. A post hoc analysis of the Group $\times$ Beam interaction (Tukey's HSD test) showed significant differences among the control and lesioned groups in all conditions except for the square-shaped broad beam. The transplanted animals, however, showed an intermediate behavior, which does not differ from that of the other lesioned groups but is also not significantly different from that of the control animals. This result confirms that uni-

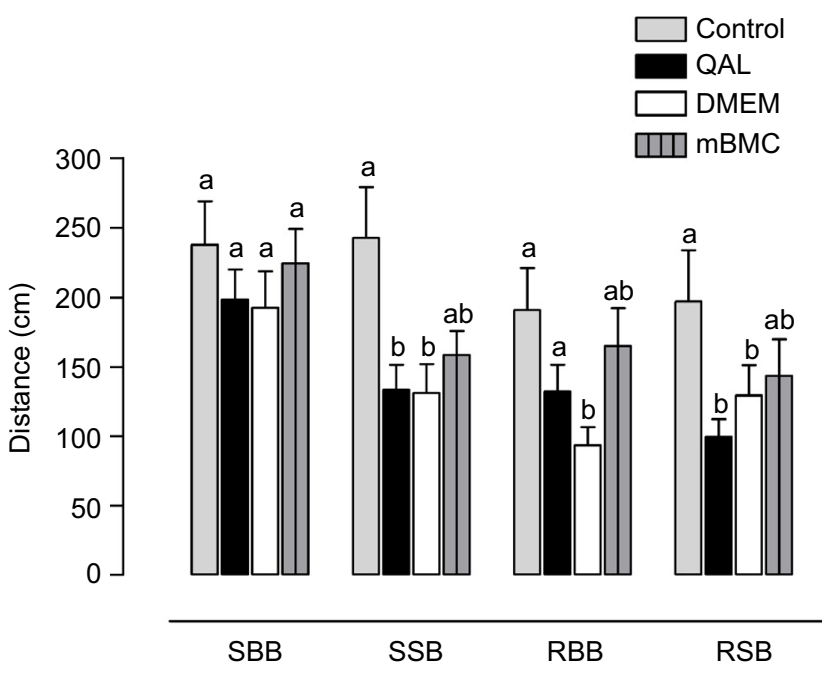

Figure 4 Results in the transversal beam test.

Notes: Test results in the 4 different types of beams: square big beam (SBB); square small beam (SSB); round big beam (RBB); and round small beam (RSB). Each column represents the distance traveled on each beam among the different experimental groups. QAL indicates the group with lesion by QA. mBMC: lesioned animals transplanted with mBMCs. QAL + DMEM: lesioned animals injected with vehicle (DMEM). Data are expressed as mean values \pm SEM (animals for each group, $n=10$ ). The letters in the upper part of the graphic indicate statistically significant differences among groups detected in the post hoc test after factorial ANOVA (Tukey's test, ${ }_{a, b} p<0.05$; different letters indicate significant differences), indicating that unilateral QA striatal lesion impairs motor coordination.

Abbreviations: ANOVA, analysis of variance; DMEM, Dulbecco's Modified Eagle's Medium; mBMC, mononuclear bone marrow cell; QA, quinolinic acid; QAL, QA lesion. lateral QA striatal lesion impairs motor coordination and suggests an effect of the transplantation in improving this motor function.

\section{Object recognition test}

On the first day, all groups spend a similar time exploring each of the 2 identical objects $\left(F_{3,41}=0.91969, p>0.05\right)$; however, lesioned animals dedicated less total time to exploration than control animals (Tukey's HSD, $p<0.05$ ). Transplanted and DMEM-treated animals showed a similar behavior as the control. On the second day (Figure 5), we found a statistically significant influence of the factor GROUP in the exploration of the new object $\left(F_{3,41}=4.84746, p<0.05\right)$. Lesioned animals explore the novel object to a significantly less extent compared to the controls and the mBMC-transplanted group (Tukey's HSD, $p<0.05)$. Vehicle (DMEM)-treated animals also show some degree of recovery in novelty recognition.

\section{Morris water maze test}

Figure 6 shows the experimental groups' performance in the spatial memory test. The escape latency showed a progressive reduction, following an asymptotical curve in all experimental groups, and no significant differences were seen among groups during acquisition $\left(F_{18,252}=1.1091\right.$, $p=0.34339$; data not shown). However, in the retention test (Figure 6), statistically significant differences

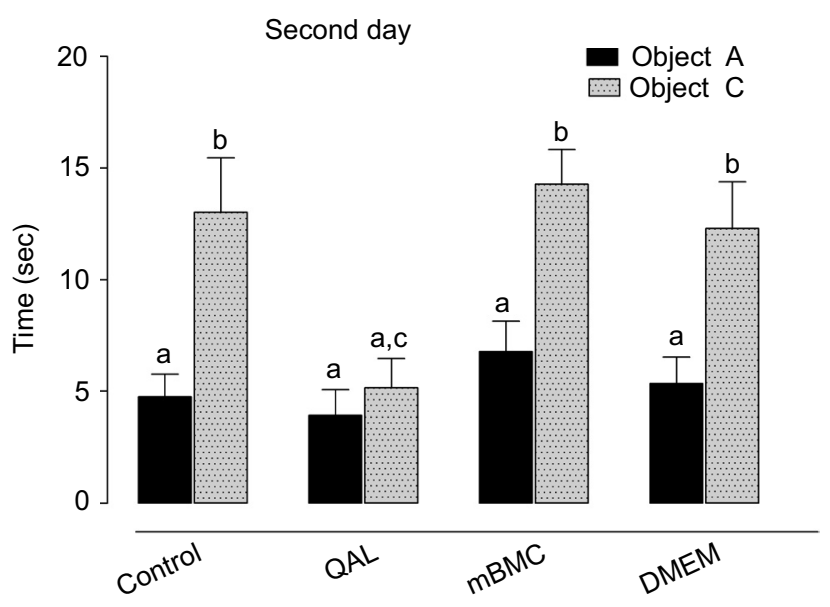

Figure 5 Results in the object recognition test.

Notes: One day after visiting the set and exploring 2 identical objects, the rats were presented with one familiar object (object $A$ ) and a novel object (object $C$ ). $\mathrm{QAL}$ indicates group with lesion from quinolinic acid. $\mathrm{mBMC}$ : lesioned animals transplanted with mBMCs. QAL + DMEM: lesioned animals injected with vehicle (DMEM). Data are expressed as mean values \pm SEM (animals for each group, $n=10$ ). The statistically significant differences correspond to post hoc test after factorial ANOVA (a-c $p<0.05$, Tukey's test); the letters in the upper part of the graphic indicate statistically significant differences among groups detected in the post hoc test after factorial ANOVA; different letters indicate significant differences.

Abbreviations: ANOVA, analysis of variance; DMEM, Dulbecco's Modified Eagle's Medium; mBMC, mononuclear bone marrow cell; QA, quinolinic acid; QAL, QA lesion; sec, seconds. 


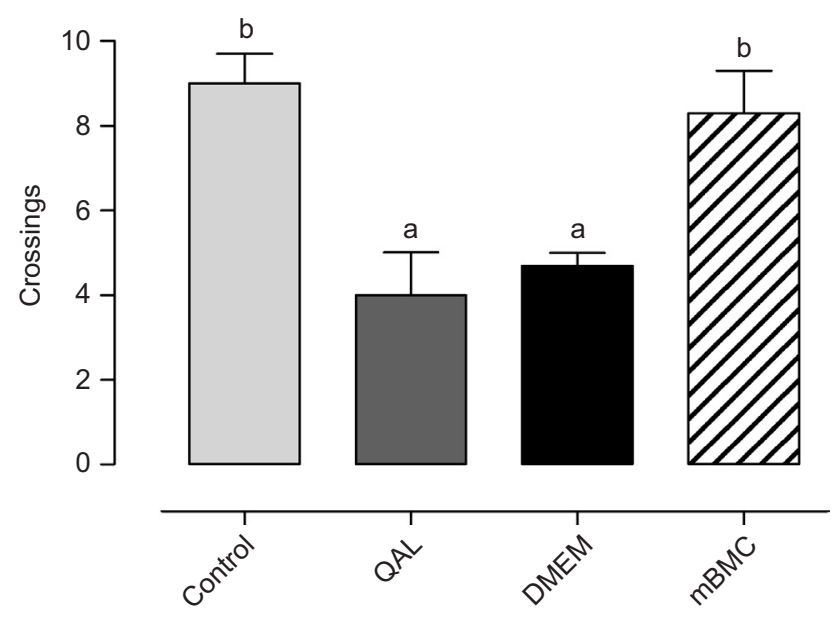

Figure 6 Morris water maze test.

Notes: Effects of $\mathrm{mBMC}$ transplantation on retention in the probe trial (without platform) in the Morris water maze test. Data are expressed as mean values \pm SEM (animals for each group, $\mathrm{n}=10$ ). The statistically significant differences correspond to post hoc test after ANOVA (a,b $p<0.05$, Tukey's test); the letters in the upper part of the graphic indicate statistically significant differences among groups detected in the post hoc test after factorial ANOVA; different letters indicate significant differences. Abbreviations: ANOVA, analysis of variance; DMEM, Dulbecco's Modified Eagle's Medium; mBMC, mononuclear bone marrow cell; $\mathrm{QA}$, quinolinic acid; $\mathrm{QAL}, \mathrm{QA}$ lesion.

$\left(F_{3,42}=9.1577, p<0.001\right)$ were found between the lesioned and DMEM-transplanted groups when compared with the mBMC-transplanted and control groups. This suggests a positive effect of transplantation on spatial memory retention.

\section{Morphology Cresyl violet}

Using a basic stain such as cresyl violet allowed a general inspection of the striatal morphology in different conditions. Figure 7A shows the normal cell distribution in this region. QA lesioning drastically reduces cell density and disrupts the pattern of cell distribution, as shown in Figure 7B, a situation that remains unchanged after DMEM injection (Figure 7C). In the transplanted striatum (Figure 7D), the cell density and distribution resemble those of the normal striatum.

\section{Immunofluorescence study by GFAP and FJC staining} Intense reactivity for GFAP was demonstrated in the striatum of lesioned and DMEM-treated animals, indicating an astrocytic gliosis in the tissue (Figure 8). Such reaction was absent in the striatum of control and transplanted animals. A similar result was seen after marking with FJC, which stains degenerating neurons. Lesioned and DMEM-treated animals showed evidence of intense degeneration, which was not seen in control and transplanted animals. DAPI was used as the marker for cell nuclei, independent of their type. In the merged panel at the right, notice the absence of GFAP and FJC in the control and transplanted animals. This result allows the assumption that transplanted cells were integrated into the host tissue.

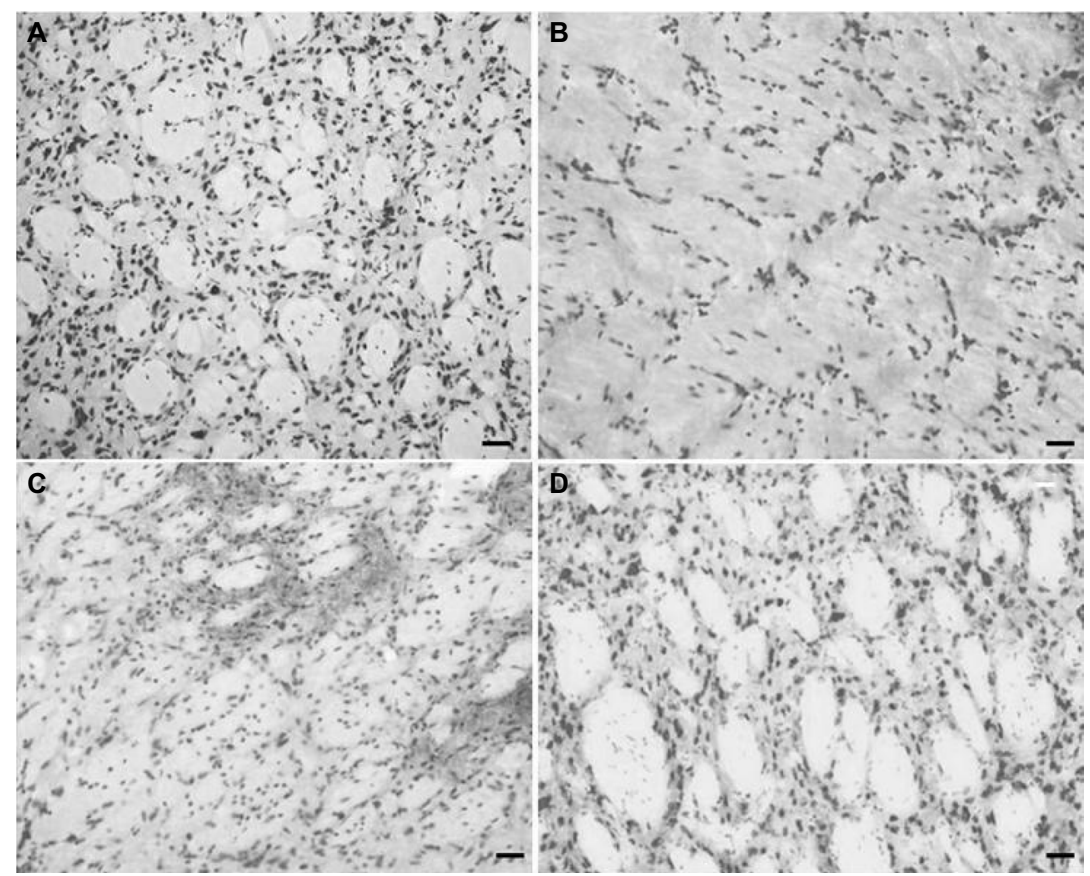

Figure 7 Basic staining with cresyl violet.

Notes: Coronal section of striatum $(25 \mu \mathrm{m})$ showing the general cytoarchitecture of the striatum in lesioned and mBMC-transplanted animals. (A) Control animal at right striatum; (B) Representative damage after QA injections into striatum showing cell loss or (C) after injection of vehicle (DMEM), to constitute a sham-transplanted group; and the striatum with $\mathrm{mBMC}$ transplantation (shown in D). Scale bars: $20 \mu \mathrm{m}$.

Abbreviations: DMEM, Dulbecco's Modified Eagle's Medium; mBMC, mononuclear bone marrow cell; QA, quinolinic acid. 


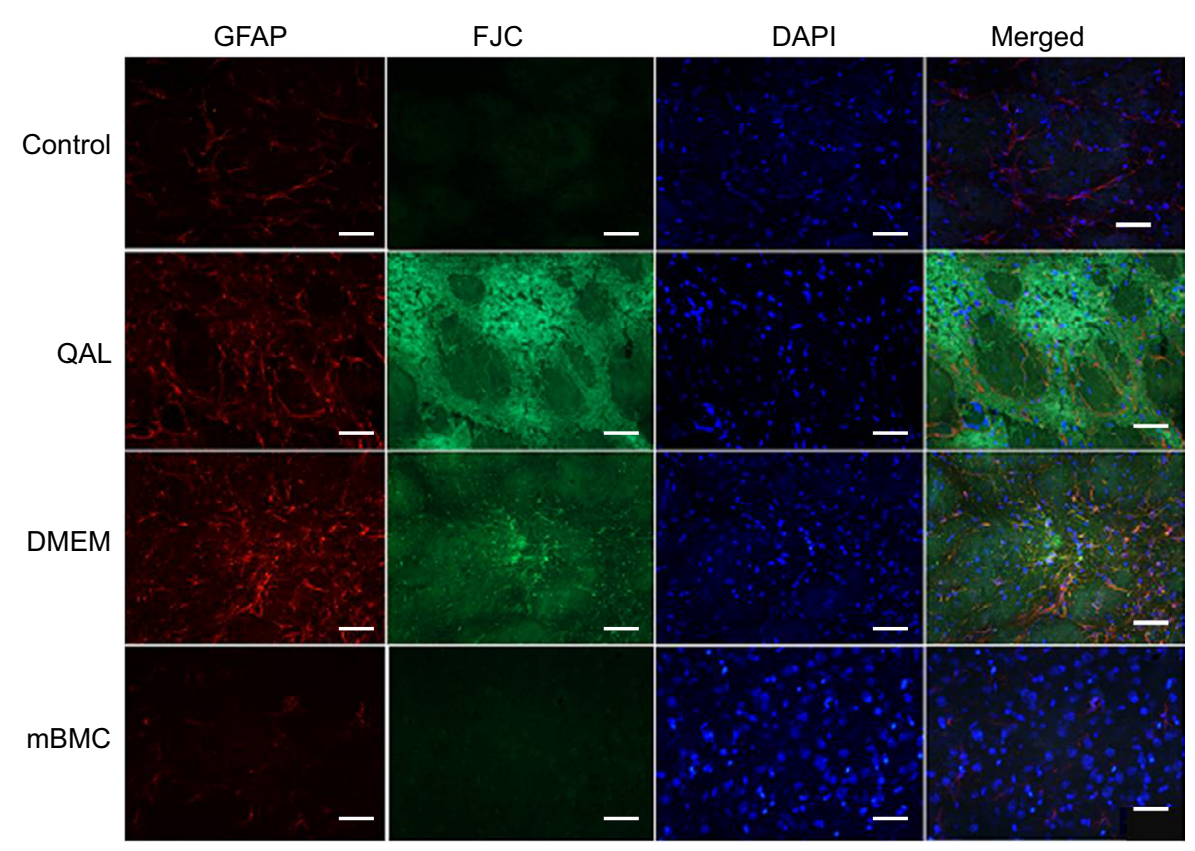

Figure 8 Immunohistochemistry study by staining with Fluoro-jade C, GFAP, and DAPI.

Notes: The rats were injected with I.2 $\mu \mathrm{L}$ of QA ( $125.5 \mathrm{nmol}$; Sigma, Saint Louis, MA, USA) in the striatum and then at 2 months postinjection they were transplanted with mBMCs or DMEM. Tissue samples were taken after completion of the behavioral tests. Scale bars: $40 \mu \mathrm{m}$. Control: nonlesioned animals. QAL group: quinolinic acid lesion. mBMC: lesioned animals transplanted with mBMCs. DMEM: lesioned animals injected with vehicle (DMEM).

Abbreviations: DAPI, 4',6-diamidino-2-phenylindole; DMEM, Dulbecco's Modified Eagle's Medium; FJC, Fluoro-Jade C; GFAP, glial fibrillary acidic protein; mBMC, mononuclear bone marrow cell; QA, quinolinic acid; QAL, QA lesion.

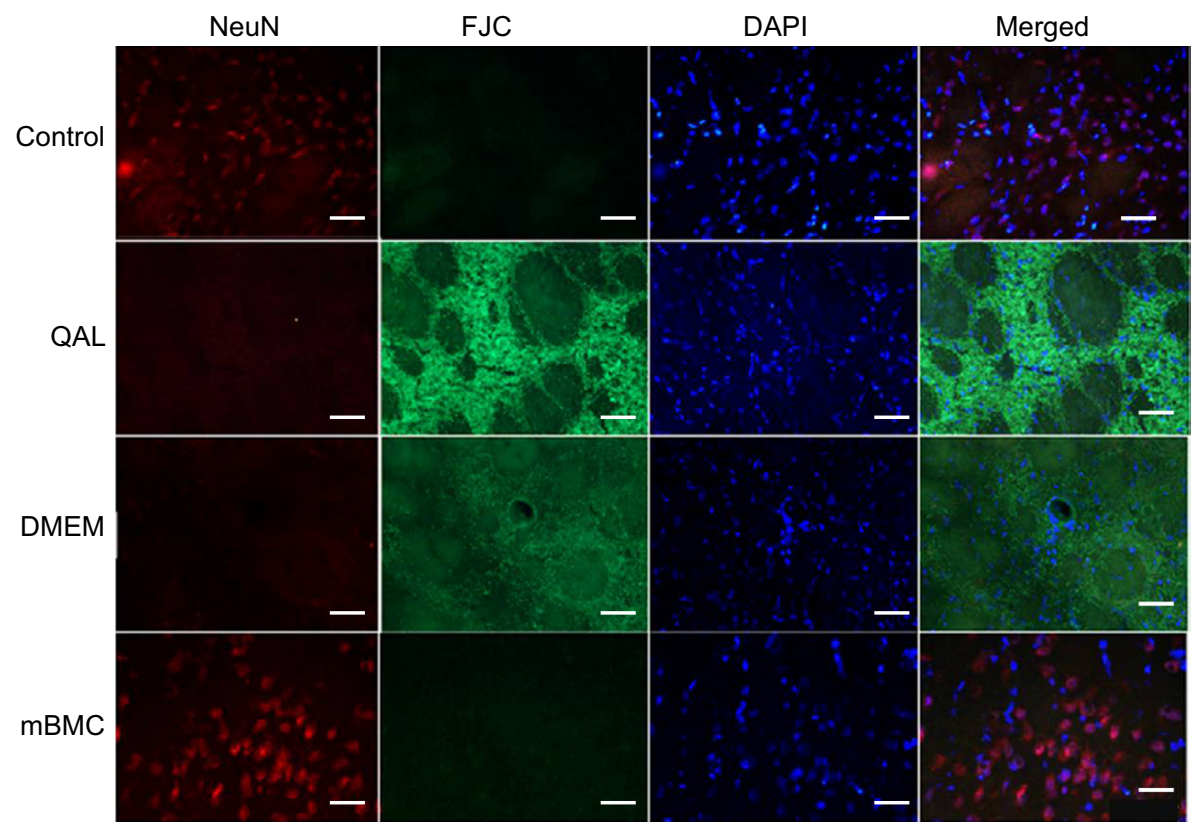

Figure 9 Immunohistochemistry study by staining with Fluoro-jade C, NeuN, and DAPI.

Notes: The rats were injected with I.2 $\mathrm{LL}$ of QA ( $125.5 \mathrm{nmol}$; Sigma, Saint Louis, MA, USA) in the striatum and then 2 months postlesion they were injected with mBMCs or DMEM. Samples were taken after completion of the behavioral tests. Scale bars: $40 \mu \mathrm{m}$. Control: nonlesioned animals. QAL group: quinolinic acid lesion. mBMC: lesioned animals transplanted with mBMCs. DMEM: lesioned animals transplanted with vehicle (DMEM).

Abbreviations: DAPI, 4',6-diamidino-2-phenylindole; DMEM, Dulbecco's Modified Eagle's Medium; FJC, Fluoro-jade C; mBMC, mononuclear bone marrow cell; NeuN, neuronal nuclear marker; QA, quinolinic acid; QAL, QA lesion.

\section{Immunofluorescence study by $\mathrm{NeuN}$ and FJC staining}

NeuN, a neuronal marker, showed positive reactivity in the control and the transplanted groups (Figure 9). In contrast, these groups were negative for FJC. The merged panel shows positive cellular bodies for NeuN in control and mBMCtransplanted groups, suggesting an integration of transplanted cells and raising even the possibility of neuronal differentiation among the transplanted cells, an aspect that we should consider in future experiments. 


\section{Cell viability and cell survival studies}

In Figure 10, we show a representative image of a sample of the cellular suspension used for transplantation marked with bisbenzimide (Hoechst 33258 reagent) to assess cell viability. The cellular viability was above $90 \%$ in all the cases.

Labeling with bisbenzimide was also used to evaluate ex vivo the survival of transplanted cells at different times after transplantation in a separate group of nontrained animals.

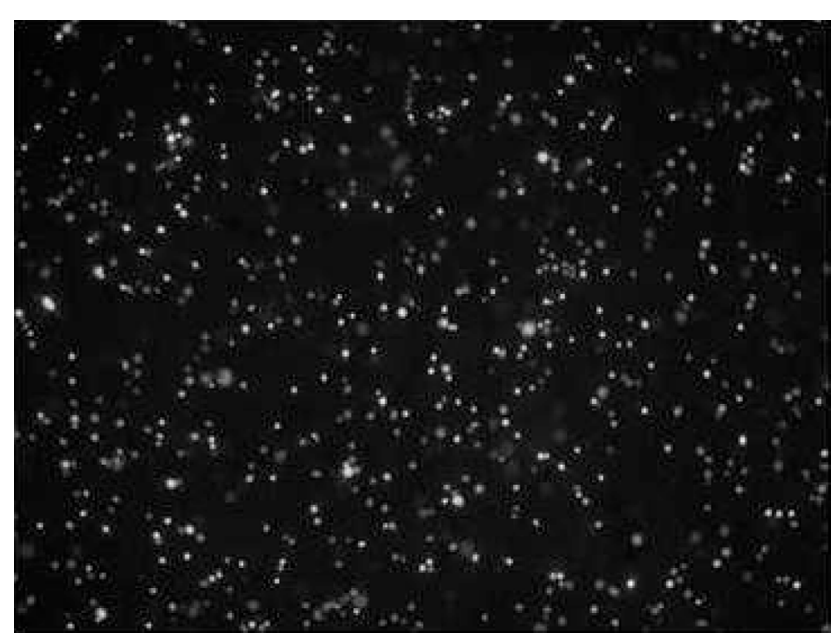

Figure 10 Viability of transplanted cells.

Notes: mBMC cells marked with bisbenzimide (Hoechst 33258 reagent, 20x) to evaluate the viability of the cells in the suspension to be used for transplantation. The evaluation was performed on an extra sample, not on the transplanted cells. Notice the presence of bright cell nuclei, indicating high viability.

Abbreviation: $\mathrm{mBMC}$, mononuclear bone marrow cell.
Figure 11 shows labeled cells at the different times that were analyzed. As can be seen, transplanted cells seemed to be integrated into the host striatal tissue and exhibited a prolonged survival (up to 1 year).

\section{Discussion}

The results of our experiments indicate that mBMCs transplanted to the striatum of QA-lesioned rats exerts a beneficial influence on the behavioral impairments caused by the lesions. QA lesioning provoked an intense glial reaction and extensive neuronal loss. These changes were not seen in transplanted animals; instead, a population of cells expressing a neuronal marker (NeuN) seems to be replenishing the striatum. It might be tempting to postulate that this population consists of neurons derived from phenotypically modified mBMCs. Such transformation has been reported in vitro, ${ }^{21,22}$ but new experiments will be required to answer this question in our in vivo model. In similarly lesioned and transplanted animals, we were able to show that transplanted cells apparently survive for a long time. Bisbenzimide might be transferred to other cells by cell fusion, but even in such a case, the transplantation procedure may still be the likely reason for the behavioral improvements.

The role of the basal ganglia in motor control and coordination is well known. Pathologies affecting its component structures, as happens in Parkinson's disease and chorea,

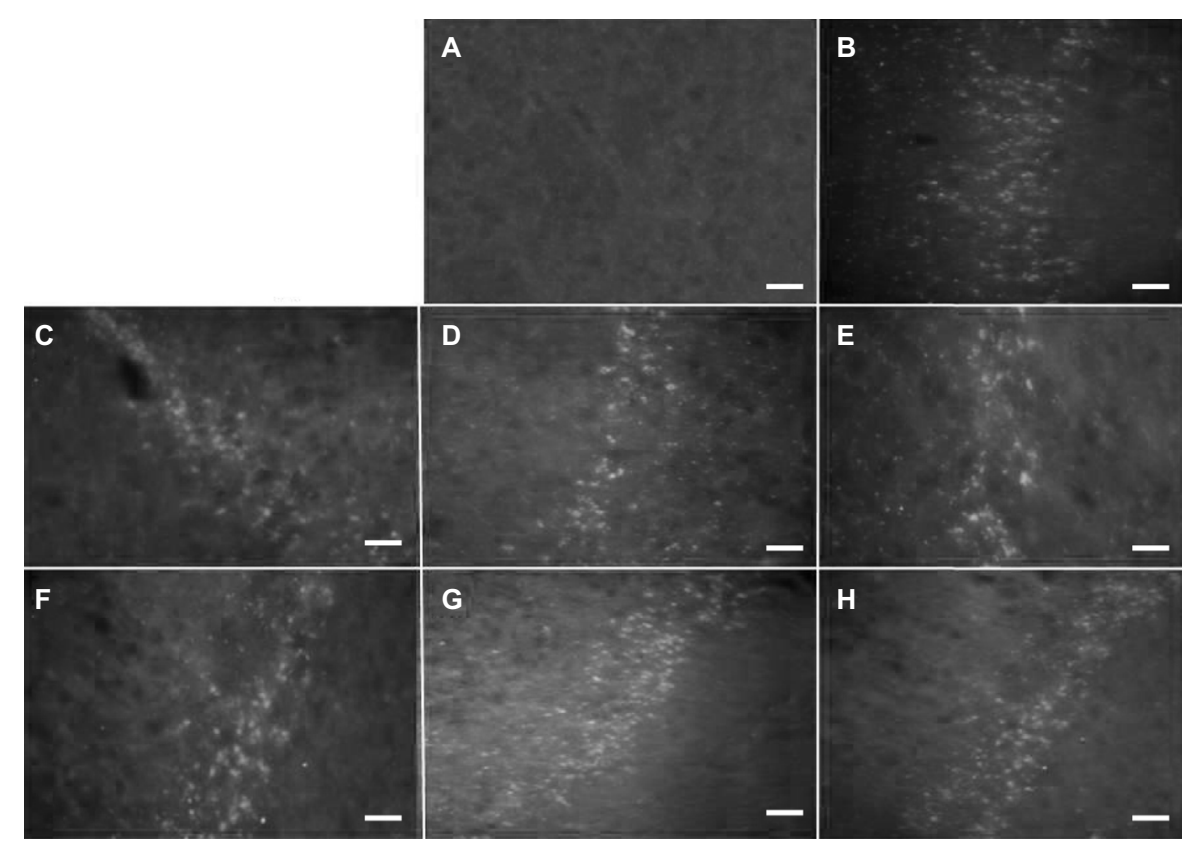

Figure I I Cell survival study.

Notes: Striatum implanted with mBMCs marked with bisbenzimide (Hoechst 33258 reagent). (A) Control striatum. (B-H) Sections from rats sacrificed I0, 30, 45, 60, 90, 180 days, and I year posttransplantation, respectively. Scale bars: $20 \mu \mathrm{m}$. The transplanted cells show durable survival.

Abbreviation: $\mathrm{mBMC}$, mononuclear bone marrow cell. 
are characterized by severe motor impairments. ${ }^{23,24}$ It is well established that QA induces the excitotoxic degeneration of medium-sized spiny striatal neurons, a pathologic landmark of Huntington's chorea. ${ }^{8}$ In animal models, the most frequently used test to measure motor impairments after lesions to the basal ganglia is the circling locomotion induced by drugs in unilaterally lesioned rats. ${ }^{25,26}$ We have previously shown this type of altered locomotion in QA-lesioned rats under amphetamine, in addition to showing that the transplantation of mBMCs reduced this abnormal behavior. ${ }^{12}$ The current paper expands those results showing that other aspects of motor function are also affected by striatal QA lesion, as is the case of the displacement and stance on the transversal beam, confirming the reports of other research groups. ${ }^{27}$

The impaired motor coordination showed by lesioned and DMEM-transplanted rats is attenuated in mBMC-transplanted rats, suggesting a restorative effect of mBMCs transplanted into the lesioned striatum in terms of motor functions.

The basal ganglia also contribute to memory functions. ${ }^{28}$ The object recognition paradigms have been reported to be affected by striatal lesions in rats. ${ }^{21}$ Our results show that QA striatal lesion reduces the exploring behavior and the identification of the novel object in the object recognition test. Transplanted animals explore significantly more and show a preference for the exploration of the novel object, suggesting a recovery of memory and visual recognition; however, the question of whether this is the result of the presence of replacement cells or an unspecific effect of injection emerges after the improvement shown by DMEM-treated animals in this test. Considering that DMEM contains high levels of aminoacids, vitamins, and glucose, it is, however, not surprising that some benefits can be derived after its local application in a lesioned tissue.

Results of transplanted animals in the Morris water maze test are consistent with the assumption that striatal lesions affect mainly procedural memory (ie, acquisition of motor skills) and not declarative or spatial memory. ${ }^{29,30}$ Nevertheless, the improved performances in the retention probe trial in the mBMC-transplanted animals suggest possible beneficial effects on this kind of memory also. We have previously shown that this transplantation increases the striatal content of BDNF. ${ }^{13}$ BDNF is a member of the neurotrophin family, which seems to act as a plasticity promoter, ${ }^{31}$ an action that may explain the memory improvements after this procedure.

The histology results in our study allow us to presume that there might be a relationship between the behavioral impairments and the histological damage to the striatum, an aspect that other authors have considered not relevant. ${ }^{32,33} \mathrm{We}$ have also provided evidence that the beneficial effect of cell therapy is associated with an apparent recovery of cells in the lesioned striatum, in particular, a population expressing a neuronal marker, in line with previous reports. ${ }^{34}$ The origin of those cells is more likely to be related to the transplanted cells, but this question should be addressed in future studies.

Other mechanisms probably involved in the restorative action of $\mathrm{mBMC}$ transplantation could be a protective action on neurons $\mathrm{s}^{35,36}$ via regulatory factors of the phosphatidylinositol 3-kinase (PI3K) signaling pathway, ${ }^{37}$ the alleged capacity of mBMCs to differentiate into a neuronal phenotype, ${ }^{7,38}$ or the induced angiogenesis and vasculogenesis required for regeneration of the damaged nerve tissue. ${ }^{39}$

\section{Conclusion}

The findings presented here confirm our previous results and those of others suggesting that transplantation with mBMCs could be a promising strategy for the treatment of Huntington's chorea. However, our findings open questions that should be addressed in future experiments. Among these are a precise quantitative morphological evaluation of the transplanted cells, their survival, proliferation, and phenotypic differentiation, to allow the quantitative study of the possible existing correlation between the morphologic changes and the behavioral improvements.

\section{Acknowledgments}

The authors thank Mei-Lee Díaz Hung for her assistance in statistical analysis. We also thank Alain García, Lorenzo Paez Tamayo, and Magalys Ramírez Figueredo for their technical support and Regina Pessoa-Pureur for her valuable contribution in the immunocytochemistry studies. This investigation was funded by the International Center for Neurological Restoration (CIREN).

\section{Disclosure}

The authors report no conflicts of interest in this work.

\section{References}

1. Sieck BC, Smith MM, Duff K, Paulsen JS, Beglinger LJ. Symptom validity test performance in the Huntington disease clinic. Arch Clin Neuropsychol. 2013;28(2):135-143.

2. Rikani AA, Choudhry Z, Choudhry AM, et al. The mechanism of degeneration of striatal neuronal subtypes in Huntington disease. Ann Neurosci. 2014;21(3):112-114.

3. Deng YP, Wong T, Wan JY, Reiner A. Differential loss of thalamostriatal and corticostriatal input to striatal projection neuron types prior to overt motor symptoms in the Q140 knock-in mouse model of Huntington's disease. Front Syst Neurosci. 2014;8:198.

4. Videnovic A. Treatment of huntington disease. Curr Treat Options Neurol. 2013;15(4):424-438.

5. Désaméricq G, Youssov K, Charles P et al. Guidelines for clinical pharmacological practices in Huntington's disease. Rev Neurol. 2016; 172(8-9):423-432. 
6. Brouillet E, Conde F, Beal MF, Hantraye P. Replicating Huntington's disease phenotype in experimental animals. Prog Neurobiol. 1999;59(5):427-468.

7. Choi KA, Hwang I, Park HS, Oh SI, Kang S, Hong S. Stem cell therapy and cellular engineering for treatment of neuronal dysfunction in Huntington's disease. Biotechnol J. 2014;9(7):882-894.

8. Beal MF, Kowall NW, Ellison DW, Mazurek MF, Swartz KJ, Martin JB. Replication of the neurochemical characteristics of Huntington's disease by quinolinic acid. Nature. 1986;321(6066):168-171.

9. Ferrante RJ, Kowall NW, Cipolloni PB, Storey E, Beal MF. Excitotoxin lesions in primates as a model for Huntington's disease: histopathologic and neurochemical characterization. Exp Neurol. 1993;119(1):46-71.

10. Mahmood A, Lu D, Yi L, Chen JL, Chopp M. Intracranial bone marrow transplantation after traumatic brain injury improving functional outcome in adult rats. $J$ Neurosurg. 2001;94(4):589-595.

11. Zhao LR, Duan WM, Reyes M, Keene CD, Verfaillie CM, Low WC. Human bone marrow stem cells exhibit neural phenotypes and ameliorate neurological deficits after grafting into the ischemic brain of rats Exp Neurol. 2002;174(1):11-20.

12. Sánchez TS, Lezcano LB, Amador EA, et al. Establishment of the conditions for the bone marrow cells transplants in a model of Huntington's disease and functional effect on the motor behavior. Rev Colomb Biotecnol. 2011;2:107-126.

13. Serrano ST, Alberti Amador E, Lorigados Pedre L, Blanco Lezcano L, Diaz Armesto I, Bergado JA. BDNF in quinolinic acid lesioned rats after bone marrow cells transplant. Neurosci Lett. 2014;559:147-151.

14. Böyum A. Separation of leukocytes from blood and bone marrow. J Clin Lab Invest. 1968;21:77-84.

15. Serrano-Sánchez T, Alberti-Amador E, Lorigados-Pedre L, íaz-Armesto ID, Blanco-Lezcano L, Vallejo-Morales A. Immunophenotype characterization of rat bone marrow cell. Rev Biotecnología Aplicada. 2005;22(3):234-236.

16. Blanco Lezcano L, Lorigados Pedre L, Ferandez Verdecia CI, Serrano Sánchez T, Pavón Fuentes N, Francis Turner L. Modify beam transversal test to evaluate hemiparkinsonian rats. Acta Biológica Colombiana. 2010;15(2):189-202.

17. Ennaceur A. One-trial object recognition in rats and mice: methodological and theoretical issues. Behav Brain Res. 2010;215(2):244-254.

18. Morris RG, Hagan JJ, Rawlins JN. Allocentric spatial learning by hippocampectomised rats: a further test of the "spatial mapping" and "working memory" theories of hippocampal function. $Q J$ Exp Psychol B. 1986;38(4):365-395.

19. Ehara A, Ueda S. Application of Fluoro-Jade C in acute and chronic neurodegeneration models: utilities and staining differences. Acta Histochem Cytochem. 2009;42(6):171-179.

20. Paxinos G, Watson C. The Rat Brain in Stereotaxic Coordinates: Hard Cover Edition. Cambridge, MA: Elsevier; 2006.

21. Soleimani M, Abbasnia E, Fathi M, Sahraei H, Fathi Y, Kaka G. The effects of low-level laser irradiation on differentiation and proliferation of human bone marrow mesenchymal stem cells into neurons and osteoblasts--an in vitro study. Lasers Med Sci. 2012;27(2):423-430.

22. Qi X, Shao M, Peng H, Bi Z, Su Z, Li H. In vitro differentiation of bone marrow stromal cells into neurons and glial cells and differential protein expression in a two-compartment bone marrow stromal cell/ neuron co-culture system. J Clin Neurosci. 2010;17(7):908-913.
23. Abada YS, Nguyen HP, Schreiber R, Ellenbroek B. Assessment of motor function, sensory motor gating and recognition memory in a novel BACHD transgenic rat model for Huntington disease. PLoS One. 2013;8(7):e68584.

24. Garcia Ruiz PJ, Gomez TE, Sanchez Bernados V, Rojo A, Fontan A, de Garcia YJ. Bradykinesia in Huntington's disease. Clin Neuropharmacol. 2000;23(1):50-52.

25. Lane EL, Cheetham SC, Jenner P. Repeated administration of the monoamine reuptake inhibitor BTS 74398 induces ipsilateral circling in the 6-hydroxydopamine lesioned rat without sensitizing motor behaviours. Eur J Neurosci. 2005;21(1):179-186.

26. Brundin P, Strecker RE, Londos E, Bjorklund A. Dopamine neurons grafted unilaterally to the nucleus accumbens affect druginduced circling and locomotion. Exp Brain Res. 1987;69(1): 183-194.

27. Bueno-Nava A, Gonzalez-Pina R, Alfaro-Rodriguez A, et al. Recovery of motor deficit, cerebellar serotonin and lipid peroxidation levels in the cortex of injured rats. Neurochem Res. 2010;35(1538):1545.

28. Havekes R, Abel T, Van der Zee EA. The cholinergic system and neostriatal memory functions. Behav Brain Res. 2011;221(2): 412-423.

29. Rossato JI, Zinn CG, Furini C, et al. A link between the hippocampal and the striatal memory systems of the brain. An Acad Bras Cienc. 2006;78(3):515-523.

30. Scimeca JM, Badre D. Striatal contributions to declarative memory retrieval. Neuron. 2012;75(3):380-392.

31. Kordower JH, Chen EY, Winkler C, et al. Grafts of EGF-responsive neural stem cells derived from GFAP-hNGF transgenic mice: trophic and tropic effects in a rodent model of Huntington's disease. $J$ Comp Neurol. 1997;387(1):96-113.

32. Bazzett T, Legnard E, Bauter MR, Albin RL. Time-course analysis and comparison of acute and chronic intrastriatal quinolinic acid administration on forelimb reaching deficits in the rat. Exp Neurol. 1999;158(1):126-134

33. Guyot MC, Hantraye P, Dolan R, Palfi S, Maziere M, Brouillet E. Quantifiable bradykinesia, gait abnormalities and Huntington's disease-like striatal lesions in rats chronically treated with 3-nitropropionic acid. Neuroscience. 1997;79(1):45-56.

34. Velloso NA, Dalmolin GD, Gomes GM, et al. Spermine improves recognition memory deficit in a rodent model of Huntington's disease. Neurobiol Learn Mem. 2009;92(4):574-580.

35. Lescaudron L. Autologous adult bone marrow stem cell transplantation in an animal model of Huntington's disease: behavioural and morphological outcomes. J Neurosci. 2003;113(7):945-956.

36. Laroni A, de Rosbo NK, Uccelli A. Mesenchymal stem cells for the treatment of neurological diseases: immunoregulation beyond neuroprotection. Immunol Lett. 2015;168(2):183-190.

37. Vivanco I, Sawyers CL. The phosphatidylinositol 3-Kinase AKT pathway in human cancer. Nat Rev Cancer. 2002;2(7):489-501.

38. Clelland CD, Barker RA, Watts C. Cell therapy in Huntington disease. Neurosurg Focus. 2008;24(3-4):E9.

39. Vaquero J, Bonilla C, Otero L, Aguayo C, Oya S, Urita M. Angiogenesis and vasculogenesis induced by intralesional adult mesenchymal stem cell transplantation in an experimental spinal cord injury model. Trauma Fund MAPFRE. 2008;19:206-211.
Journal of Neurorestoratology

\section{Publish your work in this journal}

The Journal of Neurorestoratology is an international, peer-reviewed, open access online journal publishing original research and review articles on the subject of Neurorestoratology. To provide complete coverage of this revolutionary field the Journal of Neurorestoratology will report on relevant experimental research, technological advances, and clinical achievements. The manuscript management system is completely online and includes a very quick and fair peer-review system, which is all easy to use. Visit http://www.dovepress.com/testimonials. php to read real quotes from published authors. 